\title{
3D MODELS COMPARISON OF COMPLEX SHELL IN UNDERWATER AND DRY ENVIRONMENTS
}

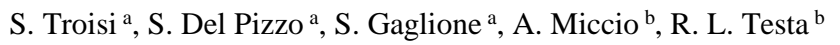 \\ a University of Naples "Parthenope", Dep. of Science and Technology, 80143 Napoli, Italy \\ (salvatore.troisi, silvio.delpizzo, salvatore.gaglione)@uniparthenope.it \\ b Protected Marine Area "Punta Campanella", Massa Lubrense, Naples, Italy - (direttore, subacquea)@ puntacampanella.org
}

Commission V

KEY WORDS: Underwater Photogrammetry, Marine Biology, 3D model, 3D comparison, Camera Calibration

\begin{abstract}
:
In marine biology the shape, morphology, texture and dimensions of the shells and organisms like sponges and gorgonians are very important parameters. For example, a particular type of gorgonian grows every year only few millimeters; this estimation was conducted without any measurement instrument but it has been provided after successive observational studies, because this organism is very fragile: the contact could compromise its structure and outliving. Non-contact measurement system has to be used to preserve such organisms: the photogrammetry is a method capable to assure high accuracy without contact. Nevertheless, the achievement of a 3D photogrammetric model of complex object (as gorgonians or particular shells) is a challenge in normal environments, either with metric camera or with consumer camera. Indeed, the successful of automatic target-less image orientation and the image matching algorithms is strictly correlated to the object texture properties and of camera calibration quality as well. In the underwater scenario, the environment conditions strongly influence the results quality; in particular, water's turbidity, the presence of suspension, flare and other optical aberrations decrease the image quality reducing the accuracy and increasing the noise on the 3D model. Furthermore, seawater density variability influences its refraction index and consequently the interior orientation camera parameters. For this reason, the camera calibration has to be performed in the same survey conditions.

In this paper, a comparison between the 3D models of a Charonia Tritonis shell are carried out through surveys conducted both in dry and underwater environments.
\end{abstract}

\section{INTRODUCTION \& MOTIVATIONS}

The Underwater Photogrammetry is a survey technique that allows the extraction of 3D model and complex measurement from a dataset of photos. This technique is widely used in archeological excavations in order to obtain a detailed documentation of the underwater archeological sites (Drap, 2012), (Bruno et al.,2013). Others applications of underwater photogrammetry have been also referred to different fields as marine biology, no-contact inspections and reverse-modeling. An innovative method of reverse-engineering for floating objects, like a boat, joins together two photogrammetric surveys: above ("dry") and below (underwater) the sea level employing special orientation devices (Menna et al., 2011); this technique has been tested during a survey of the Costa Concordia wreck providing excellent results (Menna et al., 2013). Whereas, in marine biology several interesting studies focused on marine fauna are based on the photogrammetric and computer vision techniques. In particular, the main topic of these contributions is the monitoring of marine fauna population. Stereo-vision systems are used to measure and monitor fishes (Harvey et al., 2002), (Shortis et al. 2013), and to evaluate marine organisms diversity and abundance in special areas (Cappo et al., 2003). Most operational techniques still need of a human operator, even if recent scientific publications, about the automated fish detection in underwater environment, demonstrate that, for specific purposes, an automated approach can provide good accuracies and reliability (Ravanbakhsh et al., 2015).

Underwater photogrammetry was chosen to survey the growth of Mediterranean red coral; in order to quantify the size structure of these organisms with high precision a multi-view approach was adopted (Drap et al., 2013).
Measuring in underwater environment with conventional instruments is quite complicated; the majority of marine biologists perform several visual analysis that provide measurements with low accuracy (Eleftheriou \& McIntyr, 2005). For example the chain transect is a widely used method for assessing the presence and abundance of particular species, furthermore it provides a measure of seabed relief. This is a simple and low-cost method based on a lightweight chain draped along the reef or seabed, following the contours. Measurements about a cross-section, reef composition, and an indication of reef roughness with low accuracy (precision is strictly correlated with chain link size) are obtained (Rogers et al., 1994).It is not always appropriate to measure with invasive approaches, especially when the habitats are breakable; in these cases photo-monitoring techniques are surely preferred. Generally, a housed digital camera with wide-angle lens is mounted on a metal frame known as photo-monitoring framer. The framer constrains the distance between the camera and the reef in order to obtain the same turbidity and photo illumination for each photo's dataset. The biologists repeat this acquisition more times along a given time period, therefore an accurate comparison of images from the same camera station detects transformations of the reef organisms. Of course, the accuracy of change detection is highly dependent on the precision of camera positioning as well.

Particular species grow in a single main plane and they appear completely planar. In this case, the photo-monitoring technique can be improved; indeed a framer can also provide a scale bar to perform precise measurements ensuring that the camera focal plane is parallel to the growing plane of the organism. Therefore, placing a background scale grid straight behind the organisms it is possible to carry out measurement directly on the photo. The analysis of the errors created by camera and scale- 
grid misalignment and practical methods to solve them are discussed in several contributions (Bullimore \& Hisocock, 2001).The classical measuring techniques widely employed in marine biology are inaccurate but easy to realize; this paper is going to explore the possibility to introduce a $3 \mathrm{D}$ underwater modeling as standard for marine biology purposes.

In the recent years, several low-cost software solutions have become available for the automated processing of images and the derivation of 3D models. The main processes include image orientation and dense 3D reconstruction with a high level of automation. Several critical overviews about the performance and reliability of these software were already carried out (Del Pizzo et al., 2011), (Remondino et al., 2012), (Remondino et al., 2013).

The goal of this study is to investigate the reliability results of 3D modeling in underwater environment employing an automatized procedure suitable for a non-expert photogrammetric user like a biologist. Indeed a photogrammetric survey could improve the biological classification operations: performing complex measurements on the shape, morphology and colors of marine organisms; building a global 3D database for research and educational purposes.In particular, the aim of this contribution is to detect results dissimilarity obtained by a comparison of a complex shell classical survey in "dry" and underwater conditions, analyzing the precision achieved by users with different expertise.

\section{SYSTEM SETUP}

Two different equipments were used, one for the photogrammetric survey in dry environment and another for the underwater one. In order to create a realistic scenario an expert team has realized the reference model in "dry environment", from the image acquisition for calibration procedures to the complete modeling and texturing; while the underwater survey was acquired from biologist team guided during the acquisition from the expert team.

The survey object is the biggest shell of the Mediterranean Sea, known as Triton's trumpet or Charonia Tritonis. Reaching up to $60 \mathrm{~cm}$ in length, the Charonia is a habitat for others several species of gastropods, which create complex structures on the external surface of the shell.
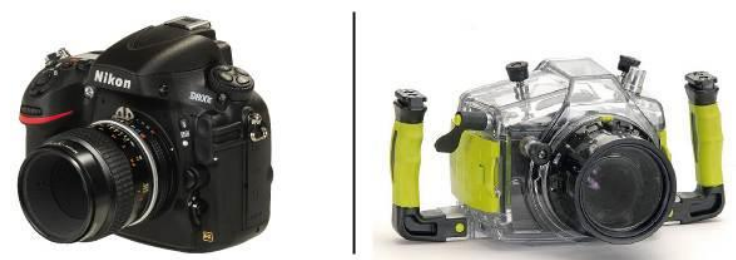

Figure 1. Cameras employed: on the left NikonD800E, on the right Nikon D7100 housed

\subsection{Equipment}

In dry environment, the equipment used for the photogrammetric survey is composed by a full frame digital camera: Nikon D800E (pixel size $4.9 \mu \mathrm{m}$ ) with $60 \mathrm{~mm}$ lens (Figure 1). Furthermore, a $100 \mathrm{~mm}$ long steel calibrated bar was used to perform an accurate $3 \mathrm{D}$ model scaling.

An ASP-C (Advanced Photo System type-C) frame digital camera: Nikon D7100 (pixel size $4.0 \mu \mathrm{m}$ ) with $60 \mathrm{~mm}$ lens was used for the underwater survey. The camera was mounted in a dedicated waterproof camera housing with a flat lens port
(Figure 1). One $150 \mathrm{~mm}$ long aluminum scale bars was also built for scaling the photogrammetric underwater measurements.

In order to obtain the camera calibration parameters, a portable volumetric rigid frame was used. It, made of aluminum, was specifically designed and build to perform self-calibrations also in underwater environment. For high accuracy and automatic measurements more than one-hundred photogrammetric circular coded targets are attached on it. The frame consists of a cross shape with four arms holding four triangular plates; it measures approximately $530 \mathrm{~mm}$ x $530 \mathrm{~mm}$ x $200 \mathrm{~mm}$.

\subsection{Calibration}

The camera calibration procedure is a fundamental task in the photogrammetric workflow. The well-known self-calibration method (Brown, 1971), (Fraser, 1997) is generally used to determine the camera calibration parameters. Generally, an inexpert end-user, who uses one of the most popular solution for automated image orientation, performs a special procedure of self-calibration indicated as "on the job" (Lhuman et al., 2007). This last procedure is not always suitable to compute correctly the interior orientation parameters; indeed a weak geometry of the network camera frame and the lack of ground control point could provide results with low accuracy and reliability. In some cases the photogrammetric model is perceptibly deformed. It has to be distinguished between image orientation and camera calibration, these are two different phases even if, in according with specific conditions, could be performed in one fell swoop.

In this contribution, both underwater and dry surveys are supported from a robust self-calibration method based on circular coded photogrammetric target (Figure 2).
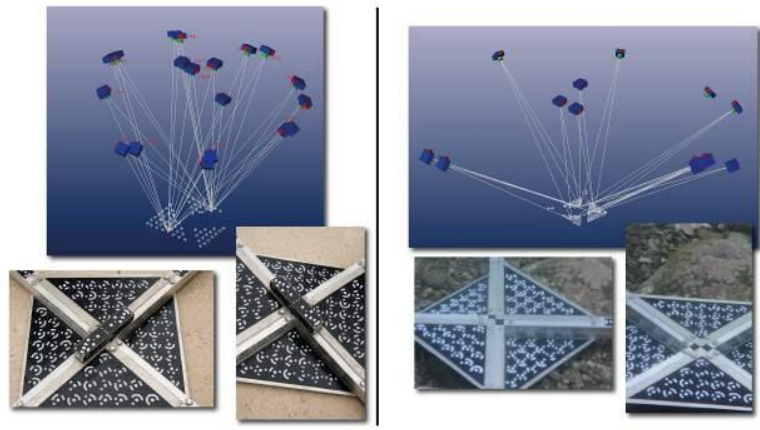

Figure 2. Camera Calibration

In order to obtain an accurate reference 3D "dry" model and therefore to preserve little particulars on shell surface, the GSD (Ground Sample Distance) was fixed to $0.05 \mathrm{~mm}$. Therefore the focal length camera was locked in according with the GSD agreed. A field of view of $35.5^{\circ}$ has been obtained mounting a $60 \mathrm{~mm}$ lens. To avoid instability on self-calibration procedure a dataset of 20 images was acquired with an average intersection angle of $77^{\circ}$. The calibration parameters obtained for the first equipment are reported in table 1 .

\begin{tabular}{|l|c|c|}
\hline \multicolumn{1}{|c|}{$\begin{array}{c}\text { Camera Calibration } \\
\text { Parameters }\end{array}$} & Value & Std Dev \\
\hline Focal length [mm] & 67.201 & 0.014 \\
Principal Point $\mathrm{x}_{0}[\mathrm{~mm}]$ & 17.881 & 0.018 \\
Principal Point y0 $[\mathrm{mm}]$ & 11.8978 & 0.018 \\
K1 $\left[\mathrm{mm}^{-2}\right]$ & $-6.797 \mathrm{e}-06$ & $6.5 \mathrm{e}-07$ \\
$\mathrm{~K} 2\left[\mathrm{~mm}^{-4}\right]$ & $-5.454 \mathrm{e}-09$ & $1.8 \mathrm{e}-09$ \\
\hline
\end{tabular}

Table 1. Nikon D800E Camera Calibration Parameters 
The same calibration procedure used for the first equipment was also applied for the calibration of the underwater equipment. The GSD was fixed at the same value of the "dry" survey, whereas the FOV value decreased to $18^{\circ}$ for both the focal length increasing and decrement of sensor width. The calibration parameters were computed from a dataset of 18 camera stations with an average intersection angle of $82^{\circ}$.

\begin{tabular}{|l|c|c|}
\hline \multicolumn{1}{|c|}{$\begin{array}{c}\text { Camera Calibration } \\
\text { Parameters }\end{array}$} & Value & Std Dev \\
\hline Focal length $[\mathrm{mm}]$ & 86.6821 & 0.175 \\
Principal Point $\mathrm{x}_{0}[\mathrm{~mm}]$ & 11.6535 & 0.032 \\
Principal Point $\mathrm{y}_{0}[\mathrm{~mm}]$ & 7.9028 & 0.077 \\
$\mathrm{~K} 1\left[\mathrm{~mm}^{-2}\right]$ & $-6.043 \mathrm{e}-005$ & $4.7 \mathrm{e}-06$ \\
$\mathrm{~K} 2\left[\mathrm{~mm}^{-4}\right]$ & $4.576 \mathrm{e}-008$ & $2.7 \mathrm{e}-08$ \\
\hline
\end{tabular}

Table 2. Nikon D7100 Camera Calibration Parameters

Table 2 shows the corresponding calibration parameters. The high value obtained for the standard deviation of the focal length can be explained by the variability of the camera station distance from the calibration device that induced the user to employ the auto-focus function on the camera to avoid blurred images.

Due to the use of the waterproof camera housing with a flat lens port, the focal length increases its value.

A flat port is essentially a flat plane of optically transparent glass or plastic in front of the lens. Flat ports are the most common lens ports used in waterproof housing for compact digital cameras. The main disadvantage of flat port is the reduction of the field of view (FOV) caused by refraction at the planar air/water interface.

Another effect causing by flat port is that objects appear larger than in air by a factor equal to, approximately, the ratio between the refraction indices of water and air.

In figure 3 are shown the radial distortion profiles for dry and underwater calibrations.
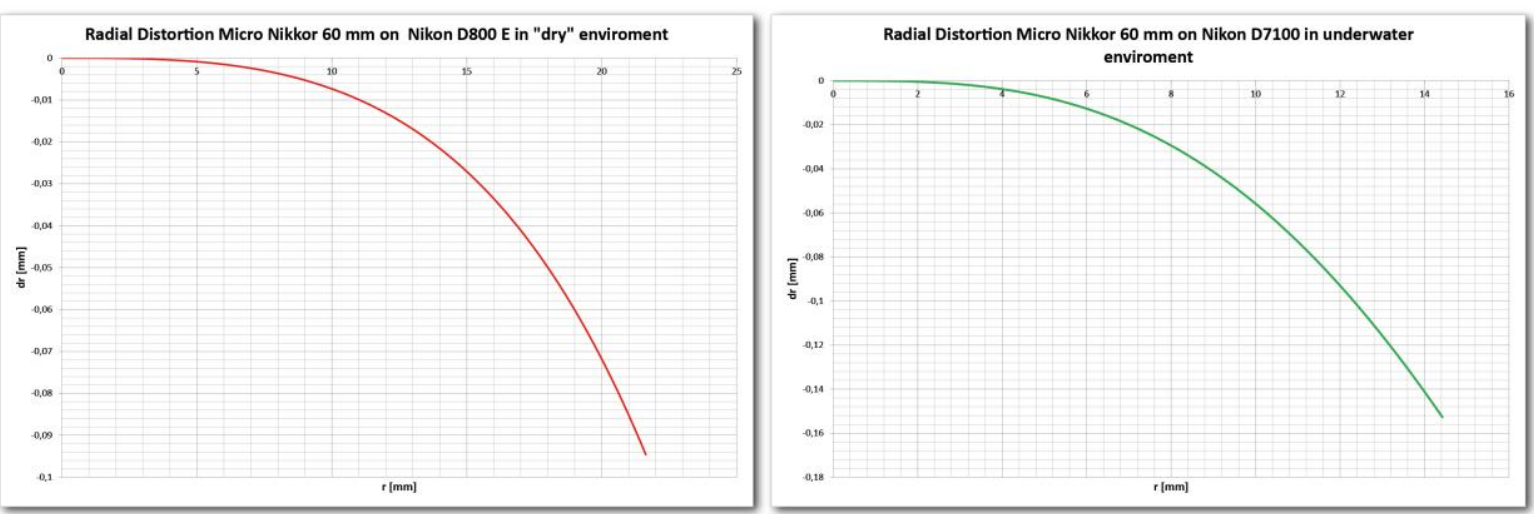

Figure 3. Radial Distortion profiles for Nikon D800E and Nikon D7100, with the same Micro Nikkor 60 mm lens, respectively in dry and underwater environments

\subsection{Surveys}

The survey is the most important phase in image-based modeling: an accurate and precise 3D model is built through a well-planned and well-realized survey. A good network geometry of camera stations allows to obtain a truthful and cleaned 3D model. Two different surveys were carried out with different problems; both in dry and underwater environments the survey operations were divided in two or more sub-surveys,

because the complex shape did not allow to achieve the whole 3D model of the shell with a single acquisition.

2.3.1 In "dry" environment: Two different sub-surveys were carried out using the same camera with the same cameraobject distance and therefore with a stable focal length. During the first acquisition, the shell was located in vertical position using a metallic support (figure 4a), a lower belt strip joints with higher strips assure a great image overlap percentage (figure 4b).

\section{(b)}
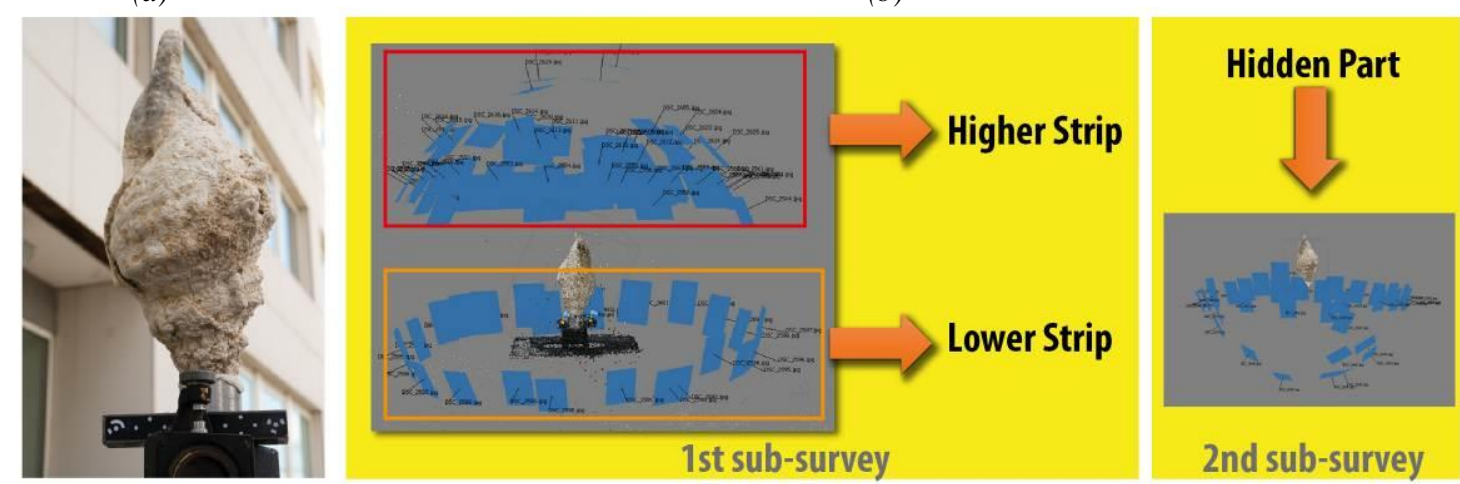

Figure 4. (a) Shell, metallic support and scale bar for the first sub-survey; (b) Network configuration of camera stations for "dry" sub-surveys. 
A set of 84 convergent images established the first acquisition, where a steel calibrated bar was employed to scale the single sub-survey. A great number of images were taken in the highest part of the shell, to assure a complete and robust coverage of rough corners.

A second acquisition, composed by 11 camera stations, was performed to survey the part of the shell hidden during the previous acquisition. In order to strengthen the connection between the two surveys, the second acquisition was planned to guarantee a great overlap zone with the first one.

In underwater environment: the survey location was chosen in order to have shallow and clear water. The shell was located in its natural habitat: rocky seabed. The presence of suspension, flare and other optical aberrations decrease the image quality and thus sensibly reduce the accuracy of point marking operations in the images, hence the precision of $3 \mathrm{D}$ point coordinates. To find the ideal light conditions for the underwater survey several tests were realized in situ at different hours of the day. The afternoon hours were ideal for the clarity and brightness of the water; a beach umbrella was used to avoid sunlight reflections on the sea surface and on the shell. A black panel was located as background under the shell, such strategy has considerably simplified the modeling operations (figure 5a). Finally, several sub-survey were carried out to obtain a complete 3D model of the shell, employing 300 camera stations. Furthermore, to simplify the survey operations the auto-focus camera was enable, even if the diver surveyor was been guided to hold a constant distance from the shell to avoid great variation in focal length. Of course, letting enable the autofocus camera the internal orientation parameters continually change, but this was preferred to blurred images.

The underwater shots were taken according to a close -range photogrammetric survey (figure $5 b$ ). (a)

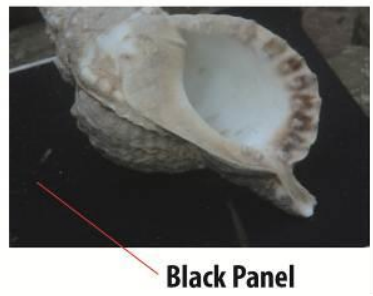

(b)

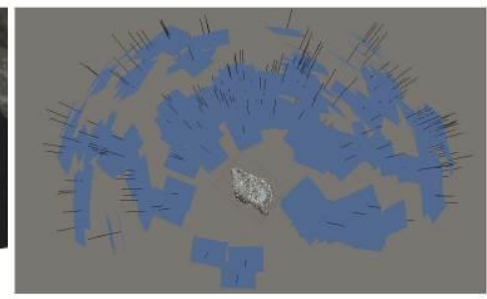

Figure 5. (a) Black panel used as background, (b) Network configuration of camera station for "underwater" survey

\section{IMAGE-BASED MODELING}

In photogrammetry the term "modeling" generally indicates the procedure that allows to gather a 3D digital model using a dataset of images. Such procedure can be divided in three fundamental steps:

1. Image orientation, that is the process able to derive the exterior (and eventually interior) parameters of each camera station. This procedure requires a reliable set of image correspondences (tie-points), which are the main input for a non-linear least square minimization;

2. Image matching: a method to compute a dense point cloud. Such task needs a precise set of oriented images as input;

3. Meshing, that collects all the algorithms employed to wrap a point cloud with a triangular mesh or mathematical surface.
In this paper the description of meshing operations has been omitted because does not provide any contribution to the inspection while first two steps were realized with Photoscan software.

\subsection{Image Orientation}

Each survey was oriented using an automatic tie point extraction operator (Lowe, 1999), (Bay et al., 2006), that provides highly distinctive features invariant to image scaling and rotations and partial invariant to brightness. Once the image correspondences are extracted, their 3D (object) coordinates are computed by means of a bundle adjustment method.

3.1.1 In "dry" environment: Each different sub-surveys was oriented independently using camera parameters computed during the calibration task.

For the first sub-survey, the software was able to orient a dataset of 84 camera stations; the calibrated bar was used to scaling the photogrammetric model.

In order to have an overlap of images and tie-points the second sub-survey was oriented using 35 images, therefore 24 images are in common with the first one. No scaling operations were adopted for the second sub-survey.

Finally, a merging process, based on common tie-points and camera stations, was executed for a new and complete photogrammetric model achievement with the same reference system and scale of the first one.

3.1.2 In underwater environment: A binary mask was created for each image in order to exclude the tie-points extracted on the background and to accept only those ones belonging to the shell surface. Unsuccessfully we tried to orient all images together using a single bundle adjustment procedure and employing the computed camera calibration parameters as pre-calibration. It was necessary divide the images in two different chunks: the first-one (small, only 65 images) where is present a calibrated aluminum scale bar and the second-one, (greater, 235 images) that assure a complete coverage of the entire shell surface.

It was not possible to merge the two photogrammetric models, accordingly to the "dry" computation, then two different image matching procedures were carried out obtaining two dense point clouds: one partial but scaled, the other unscaled but complete.

The two dense clouds were lead up in a unique reference frame and scale employing an ICP (Iterative Closest Points) with scale adjustment procedure. Once scaled, the most complete dense point cloud was used for comparing operations.

\subsection{Dense Cloud Point generation}

For both underwater and "dry" survey the dense imagematching module of Agisoft Photoscan was employed. Such software is a low-cost solution, but due to commercial reasons, few information about the image-matching algorithm are available (Remondino et al., 2013). The experience, the achieved results and the messages present on the command console suggest that the implemented procedure of image matching is based on stereo-matching methods; nevertheless, the results obtained from a stereo-couple are surely joint together with optimal algorithms of regularization.

The computational complexity of image matching procedures is very high, especially for the high-resolution images, hence this task takes more time to be completed. To speed up the dense point cloud generation, for both surveys, were used binary masks for each image, analogous to those previously introduced; indeed the $3 \mathrm{D}$ reconstruction is restricted to the 
only region of interest. Especially in the underwater model, the black background has facilitated the masking operations.
The final models are very dense: about 5 million of points for the underwater one and about 4 million in the "dry" case. The results obtained are shown in figure 6.
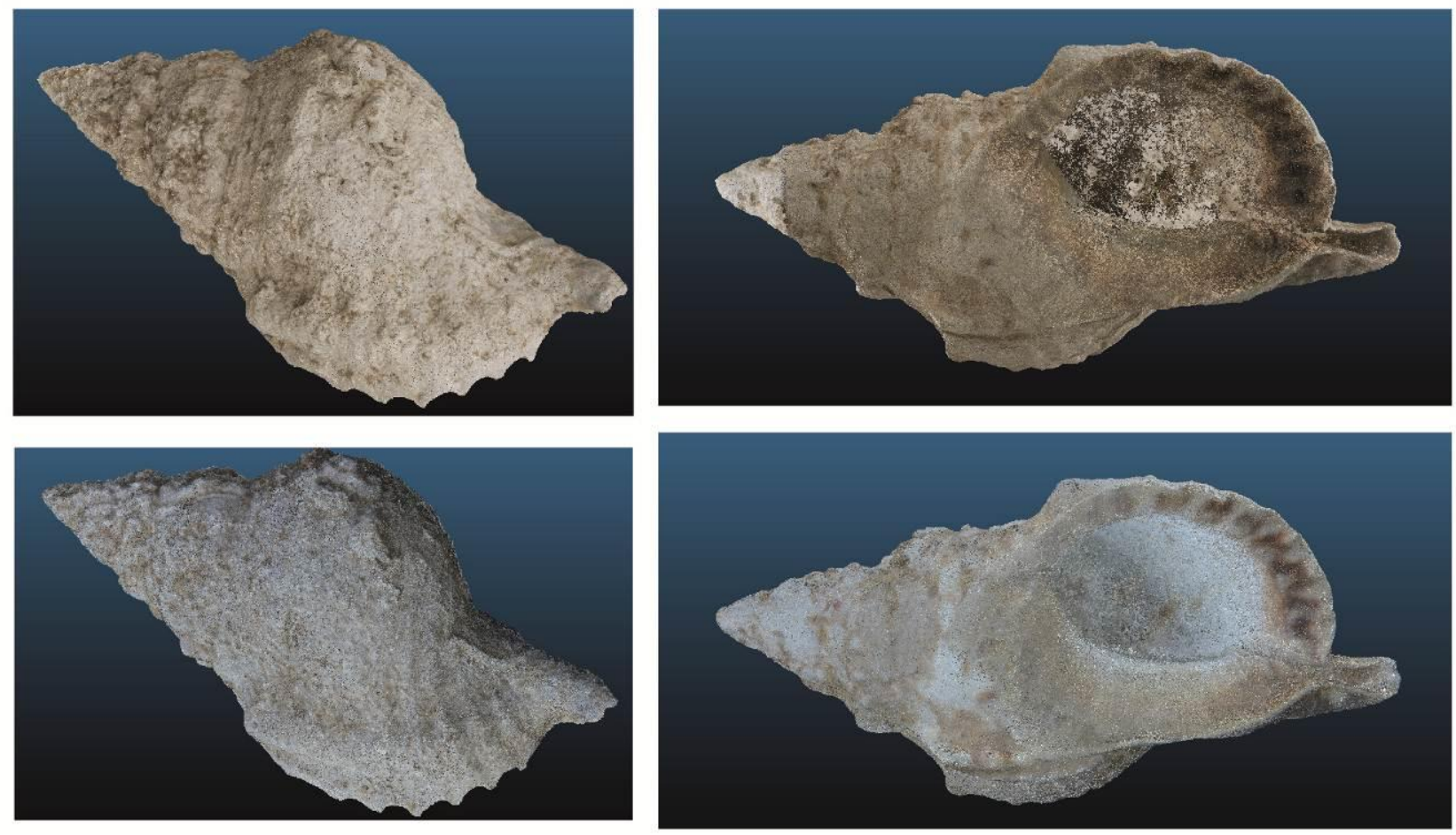

Figure 6. On the top the "dry" 3D point cloud, while in the bottom the same corresponding views of the underwater model.

\section{COMPARISON}

The output of image based modeling are two point cloud in the same scale but in different reference systems. Comparison operations can be performed only when the point cloud are located in the same frame, hence preliminary procedures has to be used in order to remove noise and to operate in a unique reference system. An open-source software named Cloud Compare was used to perform such procedures.

\subsection{Preliminary procedures}

Generally, a noise component is always present on results obtained from image-matching algorithms. To get a reliable comparison results it is necessary to detect and remove the points of the cloud identified as noise and non-connected components. Furthermore, a procedure to bring the two 3D cleaned models in the same reference system has to be applied.

4.1.1 Noise reduction: Cloud Compare offers a specific tool to detect the noise within a point cloud, based on the "octree" analysis. The same criterion for both two 3D models was applied to eliminate those not-connected components whose size where lower than a specific threshold (fixed to ten points).

The noisiest part of the point cloud was focused in the internal zone of the shell: this could be due to the reflections of the light on the very smooth parts of the internal mouth side, that works as a mirror.
4.1.2 ICP procedures: The procedure for the registration of the underwater model on the dry one was performed in two steps:

A first rough registration between the two model performed by the individuation of at least three homologous points; in this way a 7 Helmert parameters transformation is computed and applied to get roughly the target model ("underwater") on the reference one ( "dry").

To refine the rough registration the ICP algorithm (Besl \& McKay, 1992) was carried out. The algorithm iteratively revises the 7 parameters transformation needed to minimize the distance between sample points of the target model from the closest of the reference. The two steps procedure generated a final RMSE of $0.43 \mathrm{~mm}$ computed on a sample of 3000000 points chosen to minimize the above-said distances.

\subsection{Comparison \& Results}

Once the two models are in the same reference system the distance between them can be computed. Due to the complex shape of the object it is not possible employing the simple classical algorithms to compare DTM (Digital Terrain Model) or DEM (Digital Elevation Model) model, where the comparison is evaluated only in the " $z$ " directions. A plugin of "Cloud Compare" named M3C2 allows to compute signed (and robust) distances directly between two point clouds, neglecting any meshing process. A preliminary phase, that computes the normal vector for each point of two models, assures the process robustness; therefore, the distances are extracted along the normal direction to the reference model. 

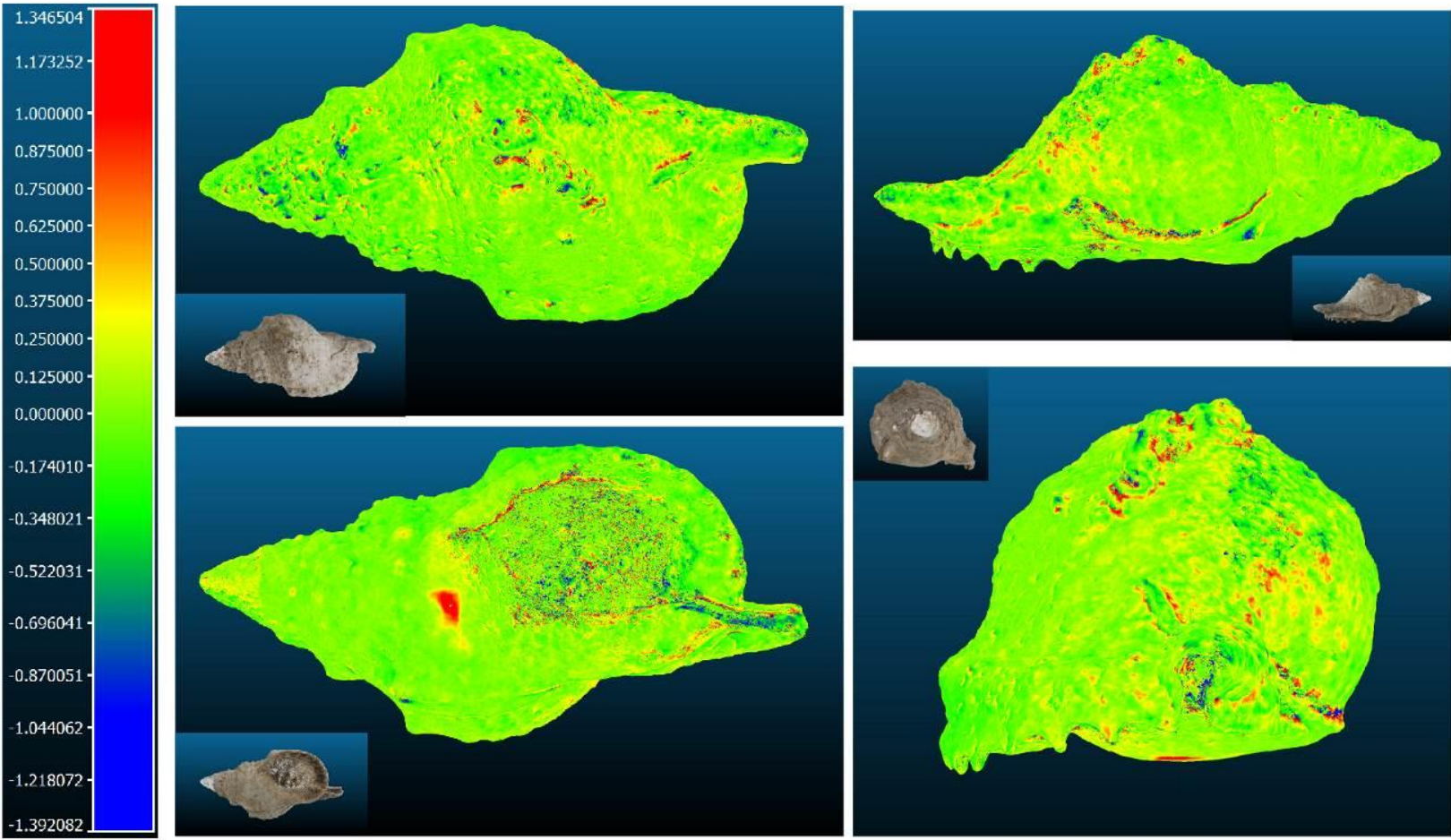

Figure 7. Meaningful views of map error between the two cloud points.

The output is a colorized texture for the reference point cloud as shown in the figure 7.

The analysis of figure 7 shows that there are no significant discrepancies between the two models, confirming that was already detectable by visual analysis downstream of modeling operations. There are only losses of minute details on the back side of the shell. Furthermore, the bottom view shows that the area of the mouth is still affected by noise despite it was submitted to a considerable job of cleaning.

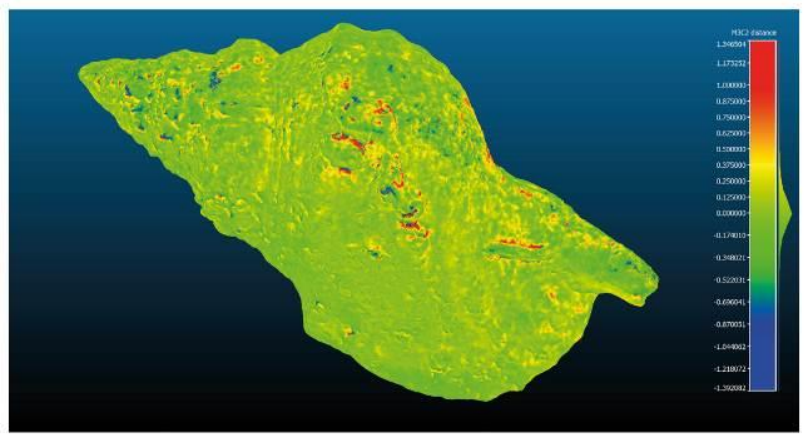

Figure 8. Error map with colored distances histogram

Figure 8 shows the color map of the differences between the models and the associated histogram. The results of statistical analysis of the differences are reported below:

- mean $=-0.0078 \mathrm{~mm}$;

- std.dev. $= \pm 1.219 \mathrm{~mm}$;

- only $5 \%$ of distances is greater than $1.23 \mathrm{~mm}$.

Such values seem very comfortable by virtue of the fact that the highest concentration of significant differences is located in the internal part of the shell.

Also figure 9, where the comparison between the two models can be investigated for given cross sections, shows that the differences are negligible for the external part of the shell. It is noteworthy to underline the benefit of cross section display that allow to measure the thickness of limestone concretions and the size of the organisms (gastropods), that live on the external surface of the shell.
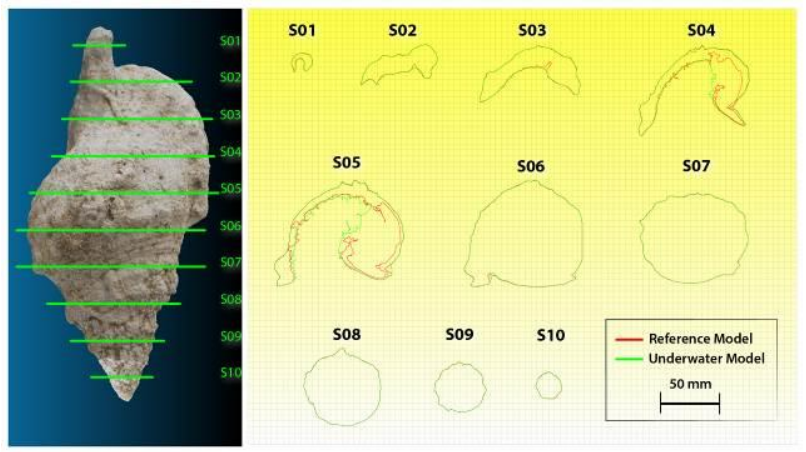

Figure 9. Cross sections comparison

Finally, in figure 10 is reported a detail of the comparison showing the effects of a small bump on the surface of the shell during transfer operations.

\section{CONCLUSION}

The results achieved from the comparison between "dry" "underwater" and photogrammetric models are very attractive mainly because that the first was obtained by an experienced user in ideal conditions while the second by a user with different expertise in non-ideal conditions.

The analysis shows that despite the inexperience and all issues related to the underwater survey the general morphology of the shell is still preserved, although some small details have been lost. 
Since a standard deviation of $1.2 \mathrm{~mm}$ of the differences between the reference dry model and the "target" one has been obtained, we can hazard that currently also a user that has few concepts of underwater photogrammetry is able to obtain measurements with millimeter accuracies and with relatively low costs equipment.

It is also to emphasize the ease of use and capacities of the software Cloud Compare that allows to process all the measurements and to analyze results, with reliability comparable with respect to commercial software.

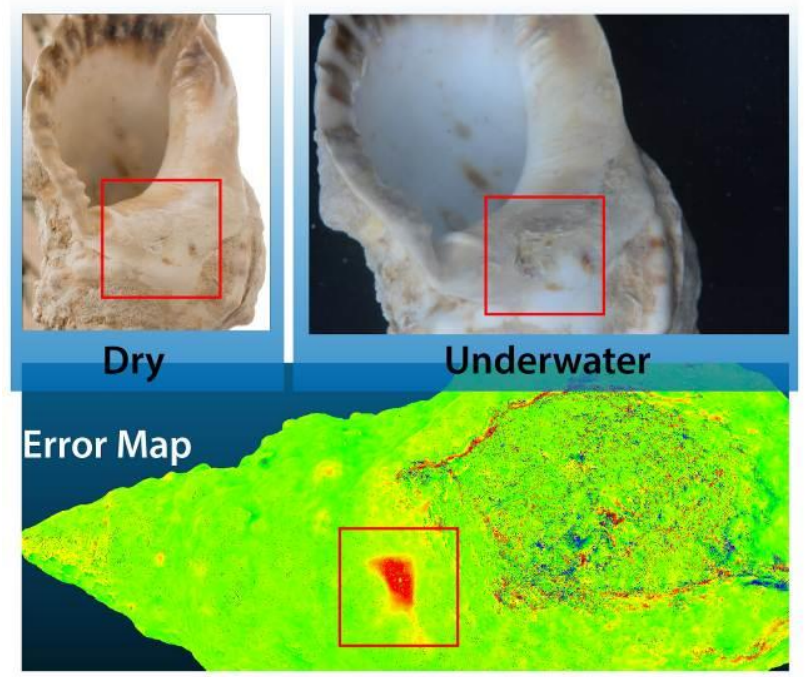

Figure 10. Detachment of a piece of shell, caused by a little incident during transfer operations.

\section{REFERENCES}

Bay, H., Tuytelaars, T., \& Van Gool, L., 2006. Surf: Speeded up robust features. In Computer vision-ECCV 2006 (pp. 404417). Springer Berlin Heidelberg.

Besl, Paul J.; N.D. McKay, 1992. A Method for Registration of 3-D Shapes. IEEE Trans. on Pattern Analysis and Machine Intelligence Los Alamitos, CA, USA: IEEE Computer Society 14 (2): 239-256. doi:10.1109/34.121791.

Brown, D.C., 1971: Close-range camera calibration. PE\&RS, Vol. 37(8), pp.855-866.

Bruno, F., Gallo, A., De Filippo, F., Muzzupappa, M., Davidde, B., Caputo P., 2013. 3D documentation and monitoring of the experimental cleaning operations in the underwater archaeological site of Baia (Italy). Proceedings of the DigitalHeritage 2013 - Federating the 19th Int'l VSMM, 10th Eurographics GCH, and 2nd UNESCO Memory of the World Conferences, Plus Special Sessions fromCAA, Arqueologica 2.0. et al. Volume 1, 2013, Article number 6743719, Pages 105112 .

Bullimore, B. \& K. Hiscock, 2001. Quantitative surveillance of sublittoral rock biotopes and species using photographs. Procedural Guideline No.3-12 In Davies, J., J. Baxter, M.Bradley, D. Connor, J. Khan, E. Murray, W. Sanderson, C. Turnbull \& M. Vincent (eds), Marine Monitoring Handbook. Joint Nature Conservation Committee.

Cappo MC, Harvey ES, Malcolm HA and Speare PJ., 2003. Potential of video techniques to monitor diversity, abundance and size of fish in studies of Marine Protected Areas. Beumer
JP, Grant A and Smith DC Aquatic Protected Areas - what works best and how do we know? World Congress on Aquatic Protected Areas proceedings, Cairns, Australia pp. 455-464.

Del Pizzo, S., Ackermann, S., Picardi, S., Troisi, S., 2011: Strumenti Open-Source per la modellazione image-based. Bollettino della società italiana di fotogrammetria e topografia 2, 25-47.

Drap, P., 2012. Underwater Photogrammetry for Archaeology. Special Applications of Photogrammetry, Dr. Daniel Carneiro Da Silva (Ed.), <http://www.intechopen.com/books/specialapplications-of-photogrammetry/underwater-photogrammetryfor-archaeology>.

Drap, P.; Merad, D.; Seinturier, J.; Mahiddine, A.; Peloso, D.; Boi, J.-M.; Long, L.; Chemisky, B.; Garrabou, J., 2013. Underwater programmetry for archaeology and marine biology: 40 years of experience in Marseille, France, Digital Heritage International Congress (DigitalHeritage). Vol.1, no., pp.97,104, doi: 10.1109/DigitalHeritage.2013.6743718.

Eleftheriou A., McIntyre A. D., 2005. Methods for the study of marine benthos, p. 418, 3rd edn. Oxford, UK: Blackwell Science.

Fraser, C. S., 1997. Digital camera self-calibration. ISPRS Journal of Photogrammetry and Remote sensing, 52(4), 149159.

Harvey, Euan; Fletcher, D.; Shortis, M., 2002: Estimation of reef fish length by divers and by stereo-video A first comparison of the accuracy and precision in the field on living fish under operational conditions. In: Fisheries Research, Vol. 57,2002 , p. 255-265.

Lowe, D. G., 1999. Object recognition from local scaleinvariant features. In Computer vision, 1999. The proceedings of the seventh IEEE international conference on (Vol. 2, pp. 1150-1157).

Luhmann, T., Robson, S., Kyle, S., \& Harley, I., 2006. Close range photogrammetry: Principles, methods and applications (pp. 1-510). Whittles.

Mark R. Shortis, Mehdi Ravanbakskh, Faisal Shaifat, Euan S. Harvey, Ajmal Mian, et al., 2013. A review of techniques for the identification and measurement of fish in underwater stereovideo image sequences", Proc. SPIE 8791, Videometrics, Range Imaging, and Applications XII; and Automated Visual Inspection, Volume 8791, doi:10.1117/12.2020941.

Menna, F., Nocerino, E., Ackermann, S., Del Pizzo, S., Scamardella, A, 2011: Underwater photogrammetry for 3D modelling of floating objects: the case study of a 19-foot motor boat. 14th Congress of Intl. Maritime Assoc. of Mediterranean IMAM 2011, Genoa, Italy.

Menna, F., Nocerino, E., Troisi, S., Remondino, F., 2013: A photogrammetric approach to survey floating and semisubmerged objects. Proc. of Videometrics, Range Imaging and Applications XII, SPIE Optical Metrology, Vol. 8791, doi: 10.1117/12.2020464.

Ravanbakhsh, M., Shortis, M. R., Shafait, F., Mian, A., Harvey, E. S. and Seager, J. W., 2015. Automated Fish Detection in 
Underwater Images Using Shape-Based Level Sets. The Photogrammetric Record, doi:10.1111.

Remondino, F., Del Pizzo, S., Kersten, T., Troisi, S., 2012: Low-cost and open-source solutions for automated image orientation - A critical overview. Proc. EuroMed 2012 Conference, M. Ioannides et al. (Eds.), LNCS 7616, pp. 40-54. Springer, Heidelberg.

Remondino, F., Spera, M.G., Nocerino, E., Menna, F., Nex, F., Gonizzi-Barsanti, S., 2013: Dense image matching: comparisons and analyses. Proc. IEEE Conference "Digital Heritage 2013", Vol. 1, pp. 47-54, 28 Oct - 1 Nov., Marseille, France.

Rogers, C. S., Garrison, G., Grober, R., Hillis, Z. M., \& Franke, M. A., 1994. Coral Reef Monitoring Manual for the Caribbean and Western Atlantic. National Park Service, Virgin Island National Park. 